\title{
Applied voltage optimization for denitrification process improvement by denitrifying species of Pseudomonas in microbial electrolysis cell (MEC)
}

\author{
Putty Ekadewi ${ }^{1}$, Matthew Hardhi ${ }^{1}$, Putri Anggun Puspitarini ${ }^{1}$, Hidayati Istiqomah $^{1}$, Cristina Gomez $^{2}$, Rita Arbianti ${ }^{1}$, \\ Tania Surya Utami $^{1 *}$, and Heri Hermansyah ${ }^{1}$ \\ ${ }^{1}$ Bioprocess Engineering Study Program, Department of Chemical Engineering, Universitas Indonesia, Depok 16424, Indonesia \\ ${ }^{2}$ Department of Bio Medicine, Indonesia International Institute for Life Sciences, Jakarta 13210, Indonesia
}

\begin{abstract}
Denitrification is the conversion process of nitrate to gaseous nitrogen forms carried out by bacteria commonly referred to as denitrifiers. Microbial Electrolysis Cell (MEC) is a type of bioelectrochemical system (BES) that is connected to external power source to aid the reactions. This research investigates the effect of applied voltage value on denitrification by nitrate removal efficiency of two model denitrifying species from the genus Pseudomonas in single-chambered MEC. Pseudomonas aeruginosa and Pseudomonas nitroreducens exhibited native removal efficiency at $70.62 \%$ and $68.20 \%$, respectively. These values respectively reached up to $89.67 \%$ and $88.58 \%$ at $1.20 \mathrm{~V}$, the upper limit of this study. Pseudomonas aeruginosa displayed better performance in MEC based off its produced current stability (mA) across the $0.35-1.20 \mathrm{~V}$ range. The effect of applied voltage on nitrate removal efficiency and setup performance was more prominent on known exoelectrogenic species of $P$ seudomonas such as $P$ seudomonas aeruginosa compared to Pseudomonas nitroreducens. Operating applied voltages of $0.35 \mathrm{~V}$ and $0.70 \mathrm{~V}$ was recommended for the application of the system based on technical and economical considerations. Further studies are needed to determine the response of the bacteria on wider range of applied voltages in MEC as well as elucidating these effects on autotrophic systems.
\end{abstract}

\section{Introduction}

Global water pollution problems have prompted the need to optimize current water treatment technologies. Nitrate-nitrogen $\left(\mathrm{NO}_{3}-\mathrm{N}\right)$ concentration is considered as one the main parameters for water pollution. US EPA have set the limit for $\mathrm{NO}_{3}-\mathrm{N}$ in water at $10 \mathrm{mg} / \mathrm{L}$, yet wastewaters that are released directly to the environment may contain up to $30 \mathrm{mg} / \mathrm{L} \mathrm{NO}-\mathrm{N}$, three times above the limit [1]. To address this problem, methodologies such as ion exchange, reverse osmosis, and electrodialysis were developed.

A drawback associated with the use of the existing technologies are closely related with environmental concerns [2]. Ion exchange generates brine high in nitrate concentration that is difficult to dispose of as well as increasing chloride concentration in the resulting effluent [3]. On the other hand, reverse osmosis and electrodialysis are both energy-intensive processes, adding to the world's energy burdens [3]. Biological methods i.e. direct denitrification by bacteria serves as an alternative to these processes. Some main advantages of denitrification over physicochemical methods were by having lower energy requirement as well as the generation of safer by-products in lesser quantity.

Denitrification is a four-step reduction process that converts nitrate into $\mathrm{N}_{2}$ gas as the final product. Although the process has been previously applied for wastewater treatment, researchers continue to put effort into improving the process. One of motives were the possible consequence of the release of one intermediate in the reaction sequence, nitrous oxide $\left(\mathrm{N}_{2} \mathrm{O}\right)$, which has been considered a potent greenhouse gas close to 300 times more harmful than carbon dioxide. The result was a heavy emphasize on the use of true denitrifiers as well as a system that allow the process to proceed through all four sequential reaction steps. Thus, most of the previous studies were focused on isolating these true and potent denitrifiers [4-6] in addition to investigating potential platform technologies for denitrification [7-9].

Bioelectrochemical systems (BES) accommodate reduction/oxidation reactions at its electrodes by the activity of microorganisms. One of its most popular configurations is microbial electrolysis cell (MEC), where the system is connected to an external power source to aid product-forming reactions that occur at the electrodes. Studies done on MEC were mostly focused on the generation of valuable chemicals such as ethanol, methane, acetate, and formic acid as products, with an important focus on biohydrogen production [10,11]. However, this system may also be used to target wastewater as a potential method for wastewater treatment.

Using BES for denitrification allows for the added value of an increased denitrification rate as well as the

Corresponding author: nana@che.ui.ac.id 
possibility to couple the system along with biohydrogen synthesis as product. Denitrification studies done on BES have shown generally positive results. An MEC based approach was done at the applied voltage of 0.80 $\mathrm{V}$ that suggested eventual slow down on nitrate removal due to substrate competition in heterotrophic system [12]. Zhu et al. [13] coupled hydrogen synthesis with denitrification aiming at improvement of autotrophic, hydrogenotrophic denitrification in a three-chambered MEC. Both studies were based on the performance of BES inoculated with mixed culture. Despite the general view that BES performed better using mixed culture, single isolate studies were also needed to better understand the use of this platform to target denitrification as a specific process.

Pseudomonads were one of the most common model organisms for denitrification studies [14-16]. Due to the abundance of pseudomonads in nature, they were commonly isolated from multiple systems including biofilms of BES, which insinuate that several strains of Pseudomonas were electroactive. Pseudomonas aeruginosa and Pseudomonas nitroreducens were regarded as denitrifiers and they have also been isolated from electroactive biofilms, which made them interesting subjects to investigate in this study $[17,18]$.

This study investigates the possibility of enhancing denitrification through the added supply of electrons via applied voltage optimization in a single chambered MEC setup. Moreover, the research specifically aims at elucidation of this effect on denitrifying bacteria commonly used as models from the Pseudomonas genus, Pseudomonas aeruginosa and Pseudomonas nitroreducens in this system grown in heterotrophic medium relying on acetate as the main organic carbon source.

\section{Materials and methods}

\subsection{Microorganism and medium}

Pseudomonas aeruginosa DSM 50071 and Pseudomonas nitroreducens IHB B 13561 were obtained from InaCC LIPI, Indonesia. The two bacterial isolates were characterized for their possession of genes central to the denitrification process, nir (nirK \& nirS) and nos $Z$ using hits obtained from NCBI gene database.

Bacterial isolates were grown on LB broth overnight at $30^{\circ} \mathrm{C}$ prior to inoculation to the MEC reactor to obtain sufficient cell density. All isolates were maintained in R2A agar plates and preserved in $40 \%$ glycerol solution at $-80^{\circ} \mathrm{C}$ for long-term storage.

Medium composition for MEC operation consisted of (in $\mathrm{g} / \mathrm{L}$, except stated) [5]: $2.7 \mathrm{~K}_{2} \mathrm{HPO}_{4}, 1.3 \mathrm{KH}_{2} \mathrm{PO}_{4}$, 0.02 yeast extract, $0.5 \mathrm{NH}_{4} \mathrm{Cl}, \quad 1.0 \mathrm{NaCl}, \quad 0.235$ $\mathrm{MgCl}_{2} \cdot 6 \mathrm{H}_{2} \mathrm{O}, 0.0795 \mathrm{CaCl}_{2} \cdot 2 \mathrm{H}_{2} \mathrm{O}, 10 \mathrm{mM} \mathrm{NaNO}, 15$

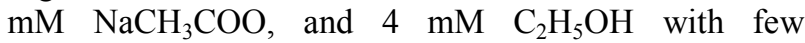
modifications from literature. Medium composition (final volume $1 \mathrm{~L}$ ) was completed with $10 \mathrm{~mL}$ of modified trace element solution [19], and $1 \mathrm{~mL}$ methylene blue ( $0.2 \%$ solution) as redox indicator [20].

\subsection{MEC setup}

The MEC setup was prepared as single-chambered miniMEC [21]. Following the previous literature, stainless steel (SS) mesh crimped with SS wire was selected to act as cathode, while isomolded graphite plate was used as anode in conjunction with grade 2 titanium (Ti) wire [21]. All electrode materials were checked for internal resistance to be under $0.50 \Omega$. MEC and experimentation setup of this study is presented in Fig. 1.

Modifications were made on the volume of study at a total of $20 \mathrm{~mL}$ as well as stopper material, which uses PTFE/silicon rubber. MEC reactors were assembled, flushed and vacuumed three times with ultra-high purity grade $\mathrm{N}_{2}$, and autoclaved. Sterile, anaerobic medium were transferred to the autoclaved MEC prior to inoculation. The volume of the medium in each MEC was $13 \mathrm{~mL}$. Bacterial inoculations from LB broth to MECs were done at $10 \%$ of medium volume. The total liquid volume in the study approached $15 \mathrm{~mL}$, leaving space for gas accumulation during the experiment.

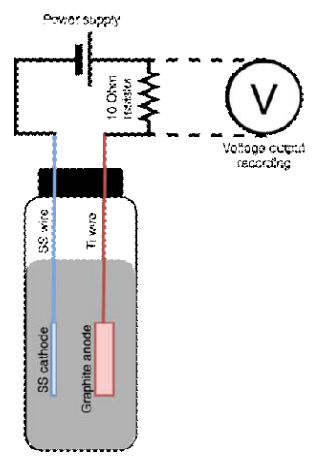

Fig. 1. MEC configuration and experimentation setup for electrical data recording in this study.

The reactors were operated in parallel at a given applied voltage value. Power supply unit (model P3005A; SUNSHINE Ltd, Guangzhou, China) was used to deliver voltages at the range of $0.35-1.20 \mathrm{~V}$. A $10 \Omega$ resistor was attached to each line connecting the power supply to the anode.

\subsection{Data collection and analysis}

Culture condition for the MEC experiment was done at room temperature without shaking. Nitrate concentration in the medium was measured periodically using UHPLC instrument, Dionex Ultimate 3000 (Thermo Fisher, USA) by passing the samples through $\mathrm{C}_{18}$ column. UHPLC analysis were modified based on Wais \& Süßmuth's protocol [22] using methanol:water mobile phase at 5:95 ratio. The flow rate was $0.300 \mathrm{~mL} / \mathrm{min}$. A UV-Vis detector was used at wavelength $240 \mathrm{~nm}$. Samples for nitrate concentration analysis were taken from the medium for a 24-hour period. Samples were pre-treated by centrifugation at 13,500 rpm for 5 minutes to separate cell mass and large particles from the supernatant. The supernatants were collected and diluted $\left(10^{-1}\right)$ using deionized water. 
Electrical measurement was done over the anoderesistor connections using digital multimeters (model AP109N; APPA Technology, Taiwan) at 10 minutes interval. Electrical output was measured for the 24-hour period in parallel with denitrification analysis. Voltage $(V)$ produced was recorded and processed using Ohm's law to obtain current $(I)[21]$.

$$
I=V / R
$$

\section{Results and discussion}

\subsection{Characterization of the microorganisms}

Anaerobic incubation setup was observed for colour changes that indicate the development of a fully reduced, anaerobic condition due to the use of methylene blue as redox indicator. These changes were observed for both Pseudomonas aeruginosa and Pseudomonas nitroreducens. Complete transparency was achieved for $P$. nitroreducens at 68 hours of incubation time, followed by $P$. aeruginos $a$ within the next 24 hours. The growth of the two pseudomonads was slower compared to their cultivation in LB broth under aerobic condition during inoculum preparation, which yielded higher turbidity at less than 24 hours incubation period. Slower growth for $P$. aeruginosa under anaerobic condition was also observed and explained by $\mathrm{Wu}$ et al. [23], largely due to the shift in protein expression.

Pseudomonas aeruginosa and Pseudomonas nitroreducens were known as some of the denitrifying species of the Pseudomonas genus [4]. However, denitrification rates among members of the Pseudomonas group was known to differ [24]. The results differentiated Pseudomonas aeruginosa from Pseudomonas nitroreducens by nitrite reductase type. Pseudomonas aeruginosa was positive for cytochrome dependent $\left(c d_{1}\right.$-NIR $)$ nitrite reductase, while Pseudomonas nitroreducens was positive for the copper dependent (CuNIR) nitrite reductase (Table 1). Both bacteria were positive for nos $Z$, which implied their ability to carry the last reaction in the denitrification reaction sequence.

Table 1. Characterization of denitrification genes in Pseudomonas aeruginosa and Pseudomonas nitroreducens.

\begin{tabular}{|c|c|c|c|}
\hline \multirow{2}{*}{ Mieroorganism } & \multicolumn{3}{|c|}{ Chy } \\
\hline & Men:-: & $m \omega$ & Win \\
\hline Preudomonas aemgina & - & . & - \\
\hline Pseudomonas nitrorctibur. & & - & - \\
\hline
\end{tabular}

\subsection{MEC Electrical Performance}

In order to act properly in BES, bacteria need the ability to transfer and utilize electrons from their environment; the ability was commonly referred to as exoelectrogenic activity or electroactive. The bacterium, Pseudomonas aeruginosa, was previously identified to possess exoelectrogenic activity [25,26]. Pseudomonas nitroreducens was previously identified as one of the community member of diesel-fed microbial fuel cell (MFC) [17]. Thus, the two bacteria were suggested to be electroactive to certain degrees.

The performance of BES was commonly assessed from its current density. Multiple studies have exhibited positive correlation between current density and applied voltage $[27,28]$. In this study it was found that initially $P$. aeruginosa had lower current generation at $0.35 \mathrm{~V}$ than $P$. nitroreducens (Fig. 2). However, at 4 hours current generation by $P$. aeruginosa managed to match $P$. nitroreducens. Current generation for $P$. aeruginosa was maintained stable above $P$. nitroreducens until the 24-hour cycle ended. At $1.20 \mathrm{~V}$ applied voltage, $P$. nitroreducens had managed to maintain higher current generation value over the 24-hour cycle yet Pseudomonas aeruginosa showed better current production based off its stability (Fig. 3).

Biomass accumulation at the electrode was observed at the anode for the two species. In comparison between the two species, $P$. nitroreducens had larger mass accumulation based on visual observation compared to $P$. aeruginosa. Nonetheless, the presence of biomass accumulation implied that these bacteria were electroactive. One mechanism that may play a role in the exoelectrogenic activity of these bacteria was their ability to secrete mediators such as pyocyanin to the medium, which helps by mediating electron transfer. Pyocyanin-mediated electron transfer mechanism was confirmed for some members of the Pseudomonas genus, particularly Pseudomonas aeruginosa $[18,25,26]$

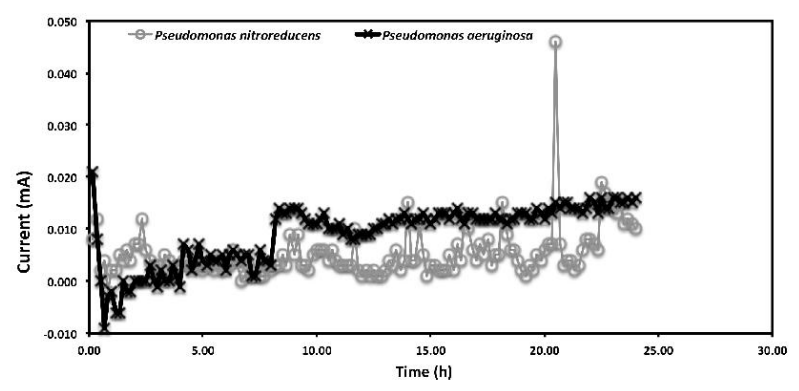

Fig. 2. Current generation from the setup at $0.35 \mathrm{~V}$ applied voltage for Pseudomonas aeruginosa and Pseudomonas nitroreducens.

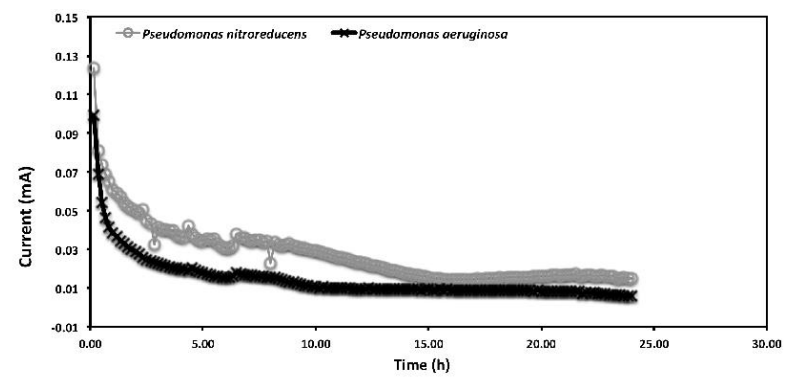

Fig. 3. Current generation from the setup at $1.20 \mathrm{~V}$ applied voltage for Pseudomonas aeruginosa and Pseudomonas nitroreducens. 


\subsection{Denitrification}

Denitrification was assessed from nitrate concentration in the medium over time as shown in Fig. 4. One of the parameters for denitrification was nitrate removal efficiency. This data was obtained by calculating the amount (in \%) of nitrate removed from the medium during reactor operation.

Nitrate concentration profile for the two bacteria was found to be similar based on the trend of the graphs. The curve for Pseudomonas nitroreducens presented sharper decline in nitrate medium concentration at the exponential growth phase between 0-4 hours. On the other hand, Pseudomonas aeruginosa exhibited significant nitrate concentration decline at later stages. Native nitrate removal efficiency for these bacteria was largely similar at $68.20 \%$ for Pseudomonas nitroreducens and $70.62 \%$ for Pseudomonas aeruginosa (Table 2.)

Optimum nitrate removal time during anaerobic incubation was a consequence of the type of NIR (nitrate reductase) enzyme that the microorganisms possess. nirK-type denitrifying bacteria had its maximum activity at the beginning of the cycle, while nirS-type had their maximum nitrate removal activity at much later time, only when the condition is fully anaerobic [29]. Characterization results confirmed Pseudomonas nitroreducens that is used in this study as nirK-type denitrifier and Pseudomonas aeruginosa as nirS-type denitrifier.

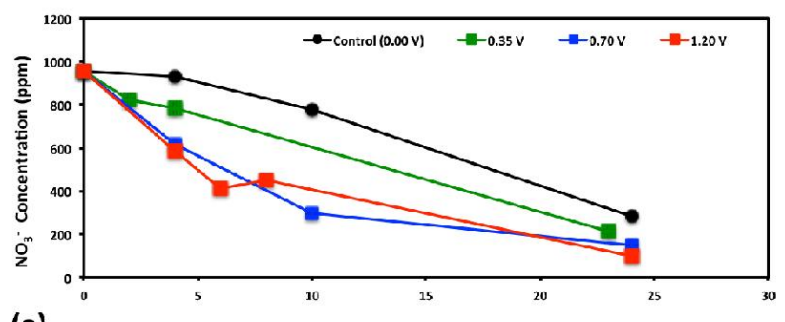

(a)

Time (h)

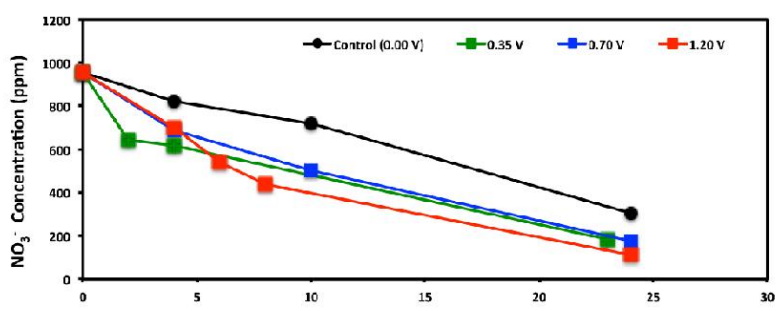

(b)

Time (h)

Fig. 4. Nitrate medium concentration over time across different values of applied voltage $(0.35,0.70$, and $1.20 \mathrm{~V})$ for (a)

Pseudomonas aeruginosa and (b) Pseudomonas nitroreducens.

End nitrate concentration was found to be consistently lowest at $1.20 \mathrm{~V}$ for both bacteria. The next best nitrate removal was at $0.70 \mathrm{~V}$ for Pseudomonas aeruginosa and $0.35 \mathrm{~V}$ for Pseudomonas nitroreducens. Based on the attained native nitrate removal efficiency and its improvements in this study, Pseudomonas aeruginosa emerge as the more promising species to be used to treat wastewater via denitrification than Pseudomonas nitroreducens.

The findings of this study presented a positive correlation between applied voltage and nitrate removal efficiency in MECs as seen in Table. 2. The maximum nitrate removal efficiency was $89.67 \%$ for Pseudomonas aeruginosa at $1.20 \mathrm{~V}$ applied voltage, followed with $88.58 \%$ for Pseudomonas nitroreducens at the same applied voltage. However, at lower applied voltage variation $(0.35 \mathrm{~V})$ Pseudomonas nitroreducens achieved better nitrate removal efficiency than Pseudomonas aeruginosa.

Table 2. Nitrate removal efficiency across different voltage values.

\begin{tabular}{|c|c|c|c|c|c|}
\hline \multirow{2}{*}{ Microorganism } & \multicolumn{4}{|c|}{ 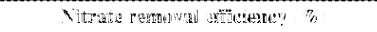 } & \multirow{2}{*}{$\begin{array}{l}\text { Jisfim } \\
\text { cumpation }\end{array}$} \\
\hline & Control & 1,356 & 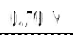 & $\therefore$ & \\
\hline Pseishomonas cierugino it & $-0+2$ & $\cdots$ & $\therefore \because$ & $1 \%$ & . \\
\hline Psendomontes nitrere theors & 582 & $\therefore \therefore$ & $\therefore^{-}$ & a & - \\
\hline
\end{tabular}

Assessment of medium nitrate concentration for each applied voltage variation is presented in Fig. 5. At 1.20 $\mathrm{V}$ the differences between Pseudomonas aeruginosa and Pseudomonas nitroreducens was smaller than at 0.35 and $0.70 \mathrm{~V}$. Near-maximum point for applied voltage improvement over nitrate removal efficiency was found at $1.20 \mathrm{~V}$. However, this study did not find the point at which no further improvement was made over the bacteria's native nitrate removal nor achieved the maximum point.

The commonly used operating value for MEC was at $<1.00 \mathrm{~V}$. This is due to several reasons, firstly bacterial cell membrane was known to be adversely impacted at the use of applied voltage above $1.00 \mathrm{~V}$ [18]. Additionally, water electrolysis cells that MEC as a platform aim to replace, normally operate at $2.00 \mathrm{~V}$. In order to maintain an advantage over water electrolysis cell, MECs were generally designed to operate at lower operating applied voltage; otherwise, MEC would lose its advantage. As a result, the operating applied voltage range for MEC was quite narrow.

In theory, the aim was to widen the gap of operating applied voltage between water electrolysis cell and MECs. For that reason, the operating values of 0.35 $\mathrm{V}$ or $0.70 \mathrm{~V}$ was more desirable than $1.20 \mathrm{~V}$ in future application of the platform although they performed below $1.20 \mathrm{~V}$ in terms of nitrate removal efficiency. 

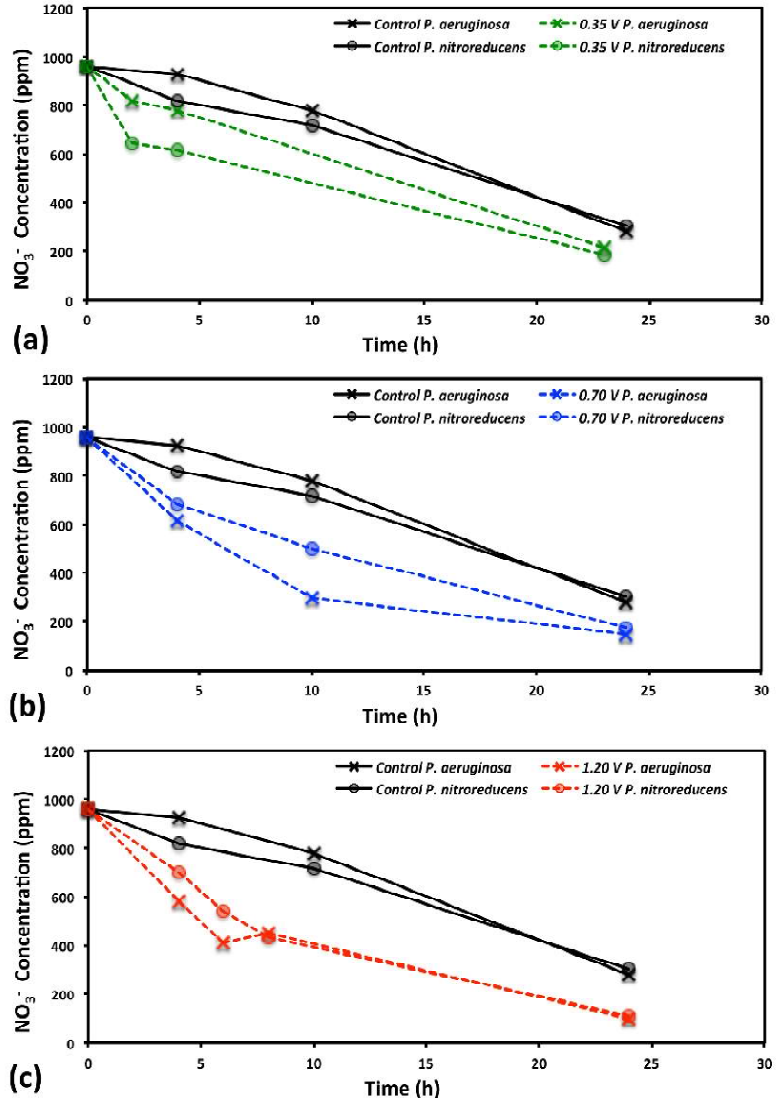

Fig. 5. Nitrate removal profile at (a) $0.35 \mathrm{~V}$ (b) $0.70 \mathrm{~V}$ and (c) $1.20 \mathrm{~V}$ against control $(0.00 \mathrm{~V})$ for Pseudomonas nitroreducens and Pseudomonas aeruginosa.

\section{Conclusions}

Pseudomonas aeruginosa and Pseudomonas nitroreducens were able to grow in the medium formulated with high nitrate concentration, close to $1,000 \mathrm{ppm}$ in this study. Native denitrification rate was shown to slightly differ between the two pseudomonads as demonstrated in the control systems. Pseudomonas aeruginosa had higher sensitivity towards voltage addition as demonstrated by the increase of its nitrate removal efficiency over each voltage variations, attaining the maximum removal efficiency at $89.67 \%$ at $1.20 \mathrm{~V}$. On the other hand, Pseudomonas nitroreducens were affected by voltage addition the most at $0.35 \mathrm{~V}$, wherein it managed to reach $80.91 \%$; higher than $P$. aeruginosa at $77.95 \%$. However, applied voltage value of $0.35 \mathrm{~V}$ and $0.70 \mathrm{~V}$ was better suited for the application of this technology based on technical and economical considerations for the operation of MEC.

Further studies are needed to evaluate the response of the bacteria towards higher applied voltage to find the maximum value at which nitrate removal efficiency cannot be further improved. Moreover, carbon source assessed in this study was limited for heterotrophic bacteria, autotrophic denitrification based off inorganic carbon source prove interesting to study in MEC, which can also be coupled with hydrogen synthesis that might better suit autotrophic or mixotrophic systems such as those commonly found in MECs.
This research was supported by Publikasi Internasional Terindeks untuk Tugas Akhir Mahasiswa UI (PITTA) research grant for the 2018 financial year.

\section{References}

[1] EPA, (2009).

[2] H. Liu, Q. Yan, W. Shen, Bioresour. Technol. 171 (2014) 187-192.

[3] J.J. Schoeman, WSA 35 (2009).

[4] K. Heylen, B. Vanparys, L. Wittebolle, W. Verstraete, N. Boon, P. De Vos, Appl. Environ. Microbiol. 72 (2006) 2637-2643.

[5] M.I. Bellini, L. Gutiérrez, S. Tarlera, A.F. Scavino, Syst. Appl. Microbiol. 36 (2013) 505516.

[6] A. Rezaee, H. Godini, S. Dehestani, S. Kaviani, 7 (2010) 313-318.

[7] A. Hussain, M. Manuel, B. Tartakovsky, J. Environ. Manag. 173 (2016) 23-33.

[8] A.K. Sahu, S.J. Ergas, Proc. Water Environ. Fed. 2005 (2005) 2183-2195.

[9] L.A. Schipper, W.D. Robertson, A.J. Gold, D.B. Jaynes, S.C. Cameron, Ecol. Eng. 36 (2010) 1532-1543.

[10] A. Kadier, M. Sahaid, P. Abdeshahian, K. Chandrasekhar, A. Mohamed, N. Farhana, W. Logroño, Y. Simayi, A. Abdul, Renew. Sustain. Energy Rev. 61 (2016) 501-525.

[11] H. Wang, Z.J. Ren, Biotechnol. Adv. 31 (2013) 1796-1807.

[12] Y. Tong, Z. He, J. Hazard. Mater. 262 (2013) 614-619.

[13] C. Zhu, H. Wang, Q. Yan, R. He, G. Zhang, Chem. Eng. J. 312 (2017) 360-366.

[14] W.G. Zumft, Microbiol. Mol. Biol. Rev. 61 (1997) 533-616.

[15] I.N. Throbäck, K. Enwall, Å. Jarvis, S. Hallin, FEMS Microbiol. Ecol. 49 (2004) 401-417.

[16] T.N. Gamble, M.R. Betlach, J.M. Tiedje, Appl. Environ. Microbiol. 33 (1977) 926-939.

[17] K. Venkidusamy, Development of Microbial Fuel Cell Technology ( MFC ) for Remediation of Hydrocarbon Contaminated Water, University of South Australia, 2012.

[18] T.C. Pannell, R.K. Goud, D.J. Schell, A.P. Borole, Biochem. Eng. J. (2016).

[19] J.P. Touzel, G. Albagnac, FEMS Microbiol. Lett. 16 (1983) 241-245.

[20] D. Karakashev, D. Galabova, I. Simeonov, World J. Microbiol. Biotechnol. 19 (2003) 233238.

[21] D.F. Call, B.E. Logan, Biosens. Bioelectron. 26 (2011) 4526-4531.

[22] S. Wais, R. Süßmuth, J. Microbiol. Methods 19 
(1994) 215-222.

[23] M. Wu, T. Guina, M. Brittnacher, H. Nguyen, J. Eng, S.I. Miller, 187 (2005) 8185-8190.

[24] C.A. Carlson, J.L. Ingraham, Appl. Environ. Microbiol. 45 (1983) 1247-1253.

[25] B.E. Logan, Microbial Fuel Cells, John Wiley \& Sons, 2008.

[26] Y. Zuo, D. Xing, J.M. Regan, B.E. Logan, Appl. Environ. Microbiol. 74 (2008) 3130-7.

[27] A. Ding, Y. Yang, G. Sun, D. Wu, Chem. Eng. J. 283 (2016) 260-265.

[28] S. Cheng, B.E. Logan, Bioresour. Technol. 102 (2011) 3571-3574.

[29] Q. Yuan, P. Liu, Y. Lu, Environ. Microbiol. Rep. 4 (2012) 113-122. 